\title{
Notification of tuberculosis: a code of practice for England and Wales
}

\author{
JOINT TUBERCULOSIS COMMITTEE OF THE BRITISH THORACIC ASSOCIATION*
}

\begin{abstract}
The notification of tuberculosis has several purposes including alerting the health services and local authorities to trace, examine, and follow up contacts and to offer other help to the patient and family. Although primarily a local control measure, reporting in addition to the Office of Population Censuses and Surveys (OPCS) enables national statistics to be compiled so that the distribution of and trends in tuberculosis may be followed over the years. Patients with tuberculosis are diagnosed and treated by doctors in a wide variety of specialties. These doctors need to be aware of the importance of notification of all their patients and of the details of the notification procedure.

A recent survey of notifications of tuberculosis in England and Wales, carried out by the Tuberculosis and Chest Diseases Unit of the Medical Research Council ${ }^{12}$ showed several ambiguities and inaccuracies in the notification procedures. To clarify the procedures and to avoid such errors, this code of practice has been prepared by the Joint Tuberculosis Committee for the guidance both of clinicians when making notifications and of medical officers for environmental health when reporting notification data to the OPCS. The recommendations take into account the revised system for submission of statistical returns by medical officers for environmental health to the OPCS introduced on 2 January $1982 .^{3}$ The Joint Tuberculosis Committee has also taken soundings from community physicians who have no adverse comments to make on the code, either as to content or practicality.
\end{abstract}

\section{Notification system in England and Wales}

A doctor who considers that a patient is suffering from tuberculosis has a statutory duty ${ }^{4}$ to notify the "proper officer" of the local authority (normally the medical officer for environmental health) using the standard notification form for notifiable infectious diseases. At the end of each week the proper officer reports to the OPCS the number of cases of tuberculosis notified in that week. The OPCS publishes the figures weekly (OPCS monitor WR), quarterly with corrections (OPCS monitor MB2), and annually (Statistics of Infectious Diseases (England and Wales) series MB2). In the revised system of submitting these statistical returns to the OPCS, ${ }^{3}$ the changes relating to tuberculosis cover the reporting of the sites of disease, cases of chemoprophylaxis, and of notifications after death. In the following sections these changes and other important problems of the notification system will be discussed, and recommendations made.

*This report was prepared by a subcommittee appointed by the Joint Tuberculosis Committee whose members were Dr K M Citron (chairman), Dr Janet Darbyshire, Professor Wallace Fox, Dr P M Lambert, Mr A J Nunn, Dr R H Raynes, Dr V H Springett, and Dr I Sutherland. It was written by Dr Citron and Dr Darbyshire.

\section{Sites of disease}

The medical officer for environmental health formerly reported cases to the OPCS as either "respiratory," "meningitis and central nervous system," or "other forms," and, owing to the lack of definition of respiratory disease, there was often confusion, particularly in cases of mediastinal lymphadenopathy or pleural effusion without a pulmonary lesion. In the new reporting system cases will be recorded as:

(1) Pulmonary lesion (with or without mediastinal nodes or pleural effusion, or both).

(2) Mediastinal nodes or pleural effusion, or both, without an identified pulmonary lesion.

(3) Meningitis (or central nervous system) alone.

(4) Other forms alone.

There is also provision for reporting separately to the OPCS patients who have lesions affecting more than one of these four groups-for example, pulmonary tuberculosis and tuberculous meningitis would be coded as " 5 ."

\section{RECOMMENDATION}

The clinician should record on the notification form all sites affected at the time of notification. The terms respiratory and non-respiratory should not be used. Pulmonary disease should be clearly differentiated from mediastinal lymphadenopathy or pleural effusions without a pulmonary lesion.

\section{Chemoprophylaxis}

Although no one would question that patients treated with chemotherapy for tuberculous disease should be notified, the position regarding cases of chemoprophylaxis is far from clear. Indeed, clinicians differ in their definition of chemoprophylaxis as well as their policy in notifying cases. Chemoprophylaxis may be defined as "the administration of one or more antituberculosis drugs to an individual, whether tuberculin negative or positive, without clinical; radiographic, or bacteriological evidence of tuberculous disease." The aim is to prevent infection in a noninfected individual or disease in an infected individual.

It is important that cases of chemoprophylaxis should not be included with notifications for tuberculous disease. The clinician, however, may wish to inform the medical officer for environmental health of such patients for the purpose of contact tracing. If so the notification form should not be used but the medical officer should be informed by letter. In the new OPCS reporting system there is provision for including cases of chemoprophylaxis in the weekly returns, and they are reported separately from the cases of tuberculosis. 


\section{RECOMMENDATION}

If the clinician wishes to inform the medical officer for environmental health of cases receiving chemoprophylaxis he should do so by letter and not by completing a standard notification form. If a patient who has chemoprophylaxis subsequently develops tuberculosis requiring chemotherapy this should be notified as a new case of tuberculosis in the usual way. Patients with a lesion on the chest radiograph, of possible activity, who are given antituberculosis chemotherapy, should be notified as cases of tuberculosis. Similarly patients treated for an illness that is suspected, on clinical grounds, to be due to tuberculosis, although not confirmed bacteriologically or radiographically, should be notified.

\section{Notifications after death}

It is important that all new cases of tuberculosis, whether diagnosed in life or after death, are notified, primarily so that local contact tracing procedures may be started but also so that the statistics are complete. Under the new OPCS reporting system notifications after death will be included in the weekly returns.

\section{RECOMMENDATION}

The clinician should notify all new cases of tuberculosis whether diagnosed in life or after death to the medical officer for environmental health on the standard notification form. If a patient has died this should be clearly indicated on the form to avoid embarrassment in the contact procedures.

Even if a doctor becomes aware only after a patient has died that the patient was suffering from tuberculosis (whether from the results of specimens collected in life or from postmortem findings) it is recommended that notification should be made on the standard form. If tuberculosis is an unexpected finding at necropsy it is recommended that the pathologist or the deceased's own general practitioner, or by local arrangement the chest physician, reports the case to the medical officer for environmental health.

In the new OPCS reporting system all new cases of tuberculosis, whether notified in life or after death, will be included in the weekly returns. In cases, however, where, by local arrangement, the registrar of deaths informs the medical officer for environmental health of a death in which tuberculosis is recorded on the death certificate, the medical officer for environmental health may need to check to see if the patient had already been notified and to contact the doctor certifying the death to find out whether the patient had active disease which should be notified.

\section{Change of diagnosis to non-tuberculous disease}

Although the clinician is not required to inform the medical officer for environmental health if the diagnosis is subsequently changed from tuberculosis to a non-tuberculous disease, it is obviously desirable that he should do so. The medical officer for environmental health should also be informed if the disease is eventually found to be due to a mycobacterium other than $M$ tuberculosis or $M$ bovis.

\section{RECOMMENDATION}

It is recommended that the clinician should inform the medical officer for environmental health whenever the diagnosis for a patient already notified is changed to a non-tuberculous disease. This should include patients in whom the disease is later found to be due to non-tuberculous mycobacteria.
All changes of diagnosis should be reported by the medical officer for environmental health to the OPCS on the quarterly correction form.

\section{Duplicate notifications and transfers}

Since 1952 local authorities have not been required to keep a register of notifications of tuberculosis. ${ }^{5}$ Without a local register, however, it has become more difficult for a doctor to discover whether or not a patient has already been notified. Furthermore, there are now no formal procedures for transferring a patient's name from one register to another when the patient moves to a different local authority area. It is the practice of some physicians to inform their authority if a patient already undergoing chemotherapy moves into the area by completing a notification form and adding the word "transfer."

\section{RECOMMENDATION}

If there is doubt whether a patient has already been notified, the clinician should make a notification-it is better to duplicate a notification than to risk its omission. The practice of completing a notification form and adding the word transfer for patients who have already been notified and are undergoing chemotherapy and who transfer to another area is to be discouraged. If further contact examinations are required in the area the medical officer for environmental health should be informed by letter.

It is recommended that the medical officer for environmental health should keep an index system of all notifications received in his area. All new notifications should be checked against this for duplicates. Cases transferred into the authority area and already notified elsewhere should not be reported to the OPCS.

\section{Renotification}

It has not been clear whether there should be renotification for patients who have had tuberculosis in the past if they develop a new episode of tuberculosis. It is important, however, that the contacts of such cases are investigated, and it is therefore recommended that there should be renotification.

\section{Summary of recommendations}

\section{THE CLINICIAN}

(1) Every effort should be made to ensure that notifications are made for all patients suffering from tuberculosis, whether diagnosed in life or after death, to the local authority on the standard notification form. If the patient has died this should be clearly stated on the form.

(2) All sites known to be affected by tuberculosis should be recorded on the notification form. The terms respiratory and non-respiratory should not be used. Pulmonary disease should be clearly identified separately from mediastinal lymphadenopathy or pleural effusion without a pulmonary lesion.

(3) Notifications for cases receiving chemoprophylaxis should not be made on the standard notification form. If the clinician wishes the medical officer for environmental health may, however, be informed of such cases by letter.

(4) Patients with disease known to be due to mycobacteria other than $M$ tuberculosis or $M$ bovis should not be notified.

(5) If the diagnosis is changed from tuberculosis to a nontuberculous disease after notification has been made the medical officer for environmental health should be informed. The isolation, after notification, of a mycobacterium other than $M$ 
tuberculosis or $M$ bovis as the causative organism should be reported as a change of diagnosis.

(6) A notification for a patient should be made if there is doubt whether one has already been made. Notifications should not be made for patients who have already been notified and who come under the clinician's care because they have changed their place of residence.

(7) If a patient who has had tuberculosis in the past develops a new episode of disease a new notification should be made.

\section{THE MEDICAL OFFICER FOR ENVIRONMENTAL HEALTH}

(1) An index system of all notifications received should be kept. Each notification received should be checked against this index to identify duplicate notifications.

(2) Notifications for patients who have had tuberculosis in the past but have developed a new episode of disease should be reported to the OPCS. If there is any doubt as to whether a second notification received for a patient is a duplicate or re- notification the medical officer for environmental health should contact the clinician to clarify the position.

(3) Any changes of diagnosis should be included on the quarterly correction form.

(4) Patients transferred into a local authority area and already notified elsewhere should not be reported to the OPCS.

\section{References}

${ }^{1}$ Medical Research Council. National survey of tuberculosis notifications in England and Wales 1978-9. Br Med F 1980;281:895.

${ }^{2}$ Davies PDO, Darbyshire J, Nunn AJ, et al. Ambiguities and inaccuracies in the notification system for tuberculosis in England and Wales. Community Medicine $1981 ; 3: 108$.

3 Office of Population Censuses and Surveys. London: OPCS, 1982. Circular (STAT), No $1 / 1982$.

${ }^{4}$ Health Services and Public Health Act 1968. London: HMSO, 1968 :ch 46, p 36.

5 The Public Health (Tuberculosis) Regulations 1952. No 704. London: HMSO, 1952: para 2.

(Accepted 23 February 1982)
A pharmaceutical house claims that its tablets are a "homoeopathic vaccine" that will "provide most people with six months' protection against colds and flu" and are "proved to be extremely effective." Each tablet is said to contain active ingredients consisting of the 30th homoeopathic strength of each of seven named influenza viruses, bacillus influenza, and bacillinum (homoeopathic). Eight tablets are to be taken on the first day and thereafter two every seven days "until course of tablets is completed." No indication is given of the number of tablets in a course. Would these claims be supported by homoeopathic physicians?

The oral influenza and common cold vaccine consists of tablets medicated with the 30th homoeopathic centesimal dilution of each of the following: influenza viruses (Asian) 1957, A 1954, and B 1954; bacillus influenza 1918; bacillinum (homoeopathic); and influenza viruses A (Hong Kong) 5/72, A (England) 42/72, and A (Port Chalmers) $1 / 73$. The usual recommended dose is two tablets, 12 hours apart in one day, repeated at fortnightly intervals. This may be continued as a prophylactic throughout the winter months, or as long as necessary. The tablets may also be used to treat colds or influenza (two or four hourly for two or three days). This treatment should not cause any side effects, and is quite widely used by patients, who often report beneficial effects. No formal clinical trials have been conducted, however.-A CAMPBELL, consultant homoeopathic physician, London.

How do steroids produce glaucoma and at what stage does it become irreversible?

The mechanism by which steroids produce glaucoma is not entirely understood, but there are three groups in the general population who differ in their ocular response to local steroids. The largest group show no change in intraocular pressure when steroid eye drops are instilled, even over a long period. In a second group, amounting to about a third of the population, prolonged application of steroid eye drops produces a moderate rise in intraocular pressure. In a third small group, comprising only about $4 \%$ of the population, there is a distinctly greater rise with a definite risk, associated with the unsupervised use of steroid eye drops, of open angle glaucoma developing at any age from infancy onwards. Patients already suffering from open angle glaucoma are made worse by local corticosteroids, and their first-degree relatives are particularly prone to develop steroidinduced glaucoma. The steroid effect is the result of a decrease of outflow of aqueous from the eye by some unspecified impairment in the trabecular meshwork, causing a type of secondary open angle glaucoma. Even in severe cases, other than those in which steroids aggravate an already existing state of glaucoma, the intraocular pressures will eventually return to normal after the steroid treatment has been terminated. By that time, however, much irreversible damage may already have been done to the sight of the eye owing to impairment of the blood supply to the optic nerve fibres by the period of high intraocular pressure. The glaucoma is irreversible once such damage has been done, but it will become stationary once the ocular hypertensive effect of the drug has ceased. Systemic steroids do not produce glaucoma in previously healthy eyes, but they too can aggravate already existing primary open angle glaucoma. It is thus essential that steroid eye drops should never be prescribed over long periods for trivial ocular irritations, and in those few cases of persistent intraocular inflammation in which their continued use is necessary there should be close long-term supervision by an ophthalmologist.-BRIAN HARCOURT, consultant ophthalmic surgeon, Leeds.

Chandler PA, Grant WM. Glaucoma. 2nd ed. Philadelphia: Lea and Febiger, 1979: 276-80.

Are the side effects of topical steroids directly related to their potency or may they be related to fluorination or other chemical formulation?

The term "flourinated steroid" refers to the presence of a fluorine atom in the 9 or 6 position, or both, of the hydrocortisone molecule. It is one of the most effective of several different chemical modifications of hydrocortisone, which has resulted in increased anti-inflammatory effect and reduced mineralocorticoid activity. Besides the steroid's intrinsic potency, the clinical effect, if topically applied, is dependent on it being absorbed into the skin, and further chemical changes to increase lipophilicity have therefore been incorporated. The concentration of the steroid, site of application, presence of occlusion, and formulation of the base also have a considerable effect on the absorption and thus on the clinical effect of these compounds. Most topical steroids have many of these chemical modifications, though there is a large variation in their resultant potency (see Mims list). The most widely used "non-fluorinated" steroid (besides hydrocortisone) is hydrocortisone butyrate, and its ranking within the potent group is well supported both experimentally and clinically. There are fluorinated compounds both more and less potent. The main debate is whether the well-publicised side effects are less pronounced with it than with other comparably potent fluorinated steroids. Some evidence suggests that it may be possible, by further chemical manipulations, to separate the wanted anti-inflammatory actions from the side effects. So far as hydrocortisone butyrate is concerned there is no question that it can cause both local atrophy and suppression of the adrenal axis, though there is a suggestion in some of the experimental and clinical reports that the side effects appear to be less than might be expected from its potency rating. It seems safest, however, to accept that no significant separation of anti-inflammatory from unwanted effects has yet been achieved and that clinical potency is directly related to the likelihood of side effects.-J A MILLAR, senior registrar in dermatology, London. 